\title{
EL FODA Y SU RELACIÓN CON LA ORGANIZACIÓN EDUCATIVA DE LA INSTITUCIÓN EDUCATIVA N³50202 “ZOILA AMORETTI DE ODRIA, DISTRITO CHAUPIMARCA
}

\author{
Mindy Cristina SUÁREZ JAVIER ${ }^{1}$
}

\begin{tabular}{lll} 
Recibido & $:$ & 26.03 .2020 \\
Aceptado & $:$ & 20.06 .2020 \\
Publicado & $:$ & 06.07 .2020 \\
\hline
\end{tabular}

RESUMEN: Esta investigación examina la relación que existe entre el FODA con la organización educativa de la Institución Educativa No 350202 "Zoila Amoretti de Odria, distrito Chaupimarca, Cerro de Pasco. El tipo de investigación fue descriptivo correlacional, bajo un diseño, no experimental transaccional de campo. La población estuvo conformada por 58 sujetos entre directivos, docentes y personal administrativo muestra para la cual se tomó el criterio de Tamayo y Tamayo. La recolección de datos se llevó a cabo a través de dos cuestionarios para los directivos y docentes, así como para el personal administrativo compuesto en escala tipo Likert, el cual fue validado por tres expertos y la confiabilidad calculada a través del coeficiente Alfa Cronbach.

Los resultados obtenidos determinaron que el FODA se relaciona eficazmente con organización educativa.

Palabras claves: FODA, gestión educativa, organización educativa, institución educativa, relación

\section{SWOT and its relationship with the educational organization of the Educational Institution $N^{o}$ 350202 "Zoila Amoretti de Odria, district Chaupimarca}

\begin{abstract}
This research examines the relationship between SWOT and the educational organization of Educational Institution No. 350202 "Zoila Amoretti de Odria, Chaupimarca District, Cerro de Pasco. The type of research was descriptive correlational, under a non-experimental transactional field design. The population consisted of 58 subjects, including principals, teachers, and administrative personnel, and the criteria used were those of Tamayo and Tamayo. Data collection was carried out through two questionnaires for principals and teachers, as well as for administrative personnel composed of a Likert scale, which was validated by three experts and the reliability calculated through the Alpha Cronbach coefficient.
\end{abstract}

The results obtained determined that SWOT is effectively related to an educational organization.

Keywords: SWOT, educational management, educational organization, educational institution, relationship.

\footnotetext{
${ }^{1}$ Universidad Nacional Mayor de San Marcos. Email: mindysuarezjavier10912@gmail.com
} 


\section{INTRODUCCIÓN}

Para el proceso de modernización de la educación a nivel nacional es necesario observar, evaluar y planificar diversas acciones que permitan dar una educación de calidad por parte de las instituciones educativas, uno de los principales objetivos de la población pasqueña, lo cual ha arribado a la investigación de este tema, dado que en su mayoría hablar de gestión de la educación nos da una amplia gama de posibilidades y variables para poder realizar nuestra investigación, lo que para los fines de este trabajo nos hemos concentrado en analizar dos variables que nos permita ganar herramientas para mejorar la gestión educativa y por ende la calidad de la educación que se le pueda brindar a los estudiantes.

Es por ello que se debe partir pro reconocer diversas fortalezas y debilidades que permitan identificar mejor los aspectos de la organización en las instituciones educativas, es por ello que identificar como el análisis de las Fortalezas, Oportunidades, Debilidades y Amenazas (en adelante FODA) se relaciona con la organización de la institución educativa N 350202 "Zoila Amoretti de Odría" ubicado en el distrito de Chaupimarca en Cerro de Pasco.

Es por ello que es necesario señalar que la importancia de investigación se basa principalmente en crear herramientas estratégicas que permitan una buena planificación educativa, lo que permita solucionar los principales problemas que generan conflictos a nivel administrativo, pedagógico e institucional.

De acuerdo al Proyecto Educativo para Pasco 2016-2021 (2015, p 57) señala claramente que debido a que la educación pública es fundamental para el desarrollo, no esperamos seguir la tendencia inercial encontrada hasta ahora en la región Pasco; sino, hacer un quiebre en la tendencia mediante la consolidación de reformas regionales, que incluyen el incremento de los fondos de inversión, la ampliación de la cobertura, el acceso y la calidad.

Así mismo en el mencionado proyecto aborda un aspecto importante: Transformar las instituciones de educación básica en organizaciones efectivas e innovadoras capaces de ofrecer una educación pertinente y de calidad, realizar el potencial de las personas y aportar al desarrollo social. Lo mencionado es una importante decisión política de generar alternativas para modernizar la educación con una visión más innovadora que permita en este caso a la institución educativa $\mathrm{N}^{\circ} 350202$ "Zoila Amoretti de Odría" no sólo mejorar la calidad de enseñanza, sino también convertirse en una institución moderna con procedimientos acordes a la demanda mundial.

\section{FODA}

Es necesario mencionar que para alcanzar los objetivos planteados es necesario comenzar con el proceso de organización y para lo cual la importancia del uso de la herramienta del FODA se volverá necesario.

Al respecto, Ramírez (2012) señala que:

Una herramienta que ayuda en el proceso de análisis de la operación de una empresa es el análisis situacional también conocido como diagnóstico FODA por sus siglas (fortalezas, oportunidades, debilidades y amenazas), ya que es la herramienta apropiada para conocer las condiciones reales de actuación de una empresa, que facilita un buen diagnóstico y evaluación en el proceso de planeación estratégica, y dada su importancia, en el presente ensayo se presenta un procedimiento para realizar un análisis FODA en una forma objetiva y de fácil aplicación para cualquier tipo de organización. (p. 54)

Lo que nos señala que es importante el uso de esta herramienta, y para el caso del sector educativo, Salas, Salas y Herrera (2011) señalan que esta debe ser una herramienta básica para la planificación, que le permitirá a la institución detectar la situación en la que se encuentra ya sea interna o externa y permitirle planificar estratégicamente asuntos a medianos, cortos o de largo plazo. Es por ello que deberá aplicarse un plan de acción en cual se aborde los siguientes puntos que se detalla a continuación. 
Journal of the Academy $|160|$

Tabla 1

Ejemplo de listado para un análisis FODA, correspondiente a una I.E en formación

\begin{tabular}{|l|l|l|l|l|}
\hline & Oportunidades & Amenazas & Fortalezas & Debilidades \\
\hline Electrónica & & $\mathrm{x}$ & & $\mathrm{x}$ \\
\hline Textil & & $\mathrm{x}$ & $\mathrm{x}$ & $\mathrm{x}$ \\
\hline Construcción & & & $\mathrm{x}$ & \\
\hline Cueros & & $\mathrm{x}$ & $\mathrm{x}$ & $\mathrm{x}$ \\
\hline Educación & $\mathrm{x}$ & & & $\mathrm{x}$ \\
\hline
\end{tabular}

Elaboración propia

Las formas metodológicas del análisis FODA varían. Una alternativa simple es un listado de las fortalezas y debilidades de la empresa, y el correspondiente listado de oportunidades y amenazas del ambiente (Power, et al. 1986).

\section{Organización Educativa}

Mientras las empresas privadas y productivas realizaban esfuerzos para mejorar su gestión, las organizaciones educativas, dependientes mayoritariamente del Estado se amparaban bajo estructuras burocráticas, que impidieron así una transformación más rápida.

Bajo la administración burocrática, las organizaciones educativas se organizaban de la siguiente manera:

- Una jerarquía o escala de mando bien determinada.

- Un sistema de procedimientos y reglas para manejar todas las eventualidades que se presenten en el trabajo.

- Una división del trabajo basada en especialización.

- Promoción y selección basada en las competencias técnicas.

- Impersonalidad en las relaciones humanas.

Sin embargo, las organizaciones educativas, sobre todo en los tiempos de cambios e incertidumbre, deben sufrir transformaciones acorde a una visión holística de la realidad con el apoyo socio-económico e histórico de la cultura a la que pertenece que ofrezca esa visión global y compleja que las organizaciones ameritan para desarrollarse y mantenerse en el tiempo, puesto que los profundos desequilibrios en la cultura involucra a la sociedad y por ende a las organizaciones educativas cuando a través de las épocas se afecta los pensamientos, sentimientos, valores, actitudes y estructuras sociales y políticas, de manera que se ha hecho prevalecer el análisis sobre la síntesis, la autoafirmación sobre la integración, la competición sobre la cooperación, la expansión sobre la conservación, y así sucesivamente hasta encontrarnos con una crisis social, moral, ecológica y espiritual de enormes dimensiones ,que ha permitido que emerjan nuevas formas paradigmáticas de mirar las instituciones y por ello establecer cambios en las organizaciones educativas. (p. 344-345).

Es para De Miguel (1989) que propuso tres indicadores para el análisis y evaluación de las organizaciones educativas:

1. Evaluación en función de objetivos

2. En relación a los Recursos

3. Indicadores de carácter social 


\section{Journal of the Academy | $161 \mid$}

\section{DESARROLLO}

\section{Material y métodos}

\section{Diseño y lugar}

El diseño de la investigación se realiza través de la observación de las variables que se han presentado y la búsqueda de su correlación, lo que nos da una investigación de carácter descriptivo-correlacional.

Asimismo, esta investigación se ha enfocado la I.E N 350202 "Zoila Amoretti de Odria del distrito Chaupimarca en Cerro de Pasco.

\section{Población}

La población de estudio se ha agrupado entre directivos, docentes y personal administrativo, un total de 58 participantes.

\section{Instrumento de recolección de datos}

Se ha realizado un cuestionario con 20 ítems, utilizando 5 alternativas en una escala Likert. Con ello se buscó recoger la percepción de las variables a las personas que se enmarcan en la investigación.

Asimismo, es necesario precisar que este instrumento fue sometido a la validez de parte de expertos en el tema, con un porcentaje de promedio de $90 \%$ de validez.

\section{$\underline{\text { Variables }}$}

\section{Variable 1: FODA}

La sigla FODA, es un acróstico de Fortalezas (factores críticos positivos con los que se cuenta), Oportunidades, (aspectos positivos que podemos aprovechar utilizando nuestras fortalezas), Debilidades, (factores críticos negativos que se deben eliminar o reducir) y Amenazas, (aspectos negativos externos que podrían obstaculizar el logro de nuestros objetivos).

López y Cano (2000) consideran que:

La técnica FODA se orienta principalmente al análisis y resolución de problemas y se lleva a cabo para identificar y analizar las Fortalezas y Debilidades de la organización, así como las Oportunidades (aprovechadas y no aprovechadas) y Amenazas reveladas por la información obtenida del contexto externo.

Dimensiones:

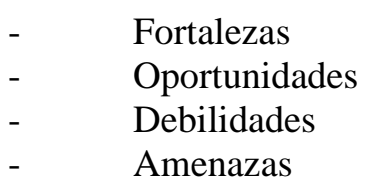

Variable 2: Organización Educativa

Es un sistema diseñado para alcanzar ciertas metas y objetivos. Estos sistemas pueden, a su vez, estar conformados por otros subsistemas relacionados que cumplen funciones específicas. 


\section{Journal of the Academy $|162|$}

Castro (2001) señala que la organización educativa, como organización autopoiética, está dada por unidades dinámicas relacionadas en una continua red de interacciones y donde se reconocen la complejidad y la incertidumbre de la realidad.

$\begin{array}{ll}- & \text { Estructura } \\ - & \text { Entorno } \\ - & \text { Proceso }\end{array}$

\section{Resultados}

FODA

TABLA N $\mathbf{N}^{\circ}$

\begin{tabular}{|l|l|l|l|l|l|}
\hline \multicolumn{2}{|c|}{1 ¿Participan todos los integrantes de la Institución en la confección de la matriz? } \\
\hline \multicolumn{2}{|c|}{} & Frecuencia & Porcentaje & $\begin{array}{l}\text { Porcentaje } \\
\text { válido }\end{array}$ & $\begin{array}{l}\text { Porcentaje } \\
\text { acumulado }\end{array}$ \\
\hline \multirow{3}{*}{ Válido } & CASI SIEMPRE & 1 & 50,0 & 50,0 & 50,0 \\
\cline { 2 - 6 } & SIEMPRE & 1 & 50,0 & 50,0 & 100,0 \\
\cline { 2 - 6 } & Total & 2 & 100,0 & 100,0 & \\
\hline
\end{tabular}

Fuente: Datos procesados mediante el software SPSS 22 y Excel.

Elaborado: Por la responsable de la investigación.

\section{GRÁFICO No 01}

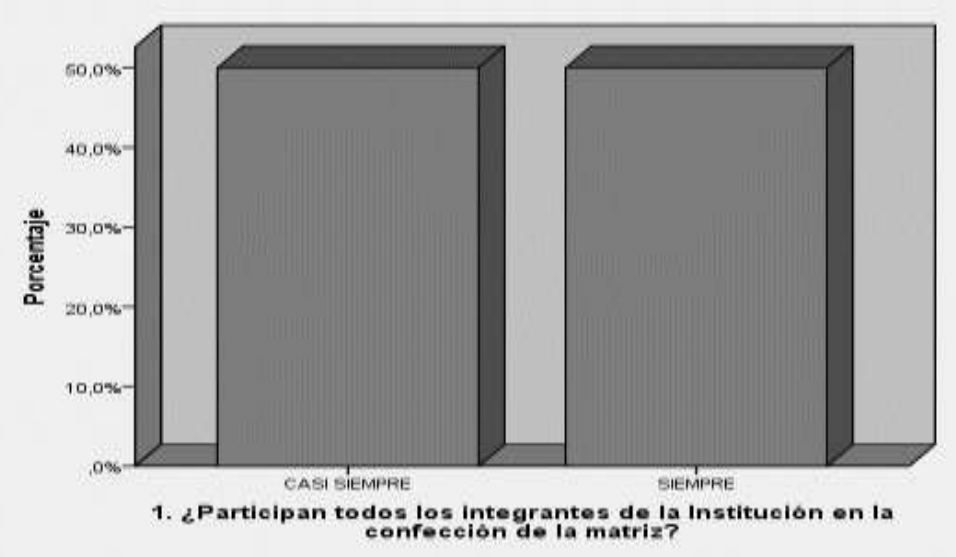

Interpretación: De acuerdo a la tabla estadística y el grafico, se evidencia un total de 2 Directivos encuestados representando que el 50,0\% manifiesta que casi siempre participan todos los integrantes de la Institución en la confección de la matriz y el 50,0\% señala que siempre.

\section{TABLA $\mathbf{N}^{\circ} 03$}

2. ¿Tiene buenas ventajas ante los demás colegios de la provincia?

\begin{tabular}{|l|l|l|l|l|l|}
\hline \multicolumn{2}{|c|}{} & Frecuencia & Porcentaje & $\begin{array}{l}\text { Porcentaje } \\
\text { válido }\end{array}$ & $\begin{array}{l}\text { Porcentaje } \\
\text { acumulado }\end{array}$ \\
\hline \multirow{3}{*}{ Válido } & A VECES & 1 & 50,0 & 50,0 & 50,0 \\
\cline { 2 - 6 } & CASI SIEMPRE & 1 & 50,0 & 50,0 & 100,0 \\
\cline { 2 - 6 } & Total & 2 & 100,0 & 100,0 & \\
\hline
\end{tabular}

Fuente: Datos procesados mediante el software SPSS 22 y Excel.

Elaborado: Por la responsable de la investigación. 


\section{Journal of the Academy $|163|$}

\section{GRÁFICO Nº2}

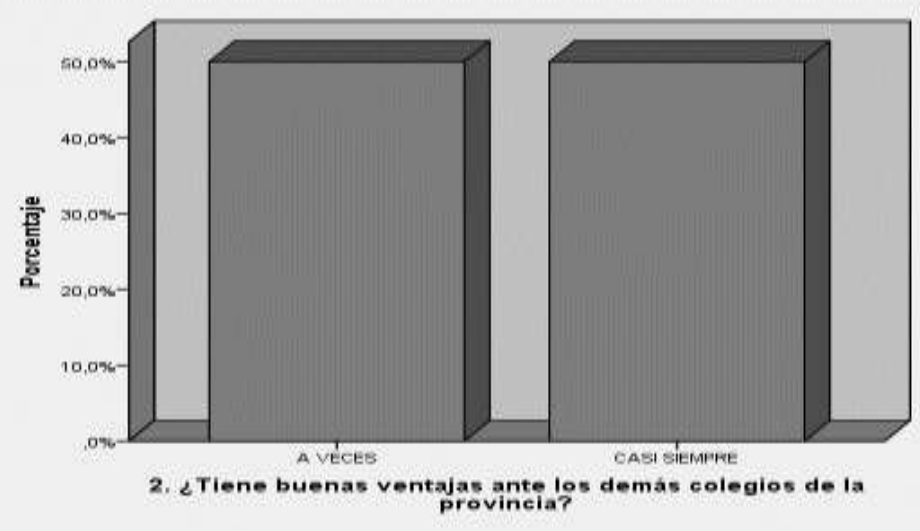

Interpretación: De acuerdo a la tabla estadística y el grafico, se evidencia un total de 2 Directivos encuestados representando que el 50,0\% manifiesta que a veces tienen buenas ventajas ante los demás colegios de la provincia y el 50,0\% señala que casi siempre.

\section{TABLA N ${ }^{\circ} 04$}

\begin{tabular}{|l|l|l|l|l|l|}
\hline \multicolumn{6}{|c|}{ 3. ¿Existen amenazas alrededor de la institución educativa? } \\
\hline \multicolumn{2}{|l|}{} & Frecuencia & Porcentaje & $\begin{array}{l}\text { Porcentaje } \\
\text { válido }\end{array}$ & $\begin{array}{l}\text { Porcentaje } \\
\text { acumulado }\end{array}$ \\
\hline Válido & CASI SIEMPRE & 2 & 100,0 & 100,0 & 100,0 \\
\hline
\end{tabular}

Fuente: Datos procesados mediante el software SPSS 22 y Excel.

Elaborado: Por la responsable de la investigación.

\section{GRÁFICO No $\mathbf{N}^{\circ}$}

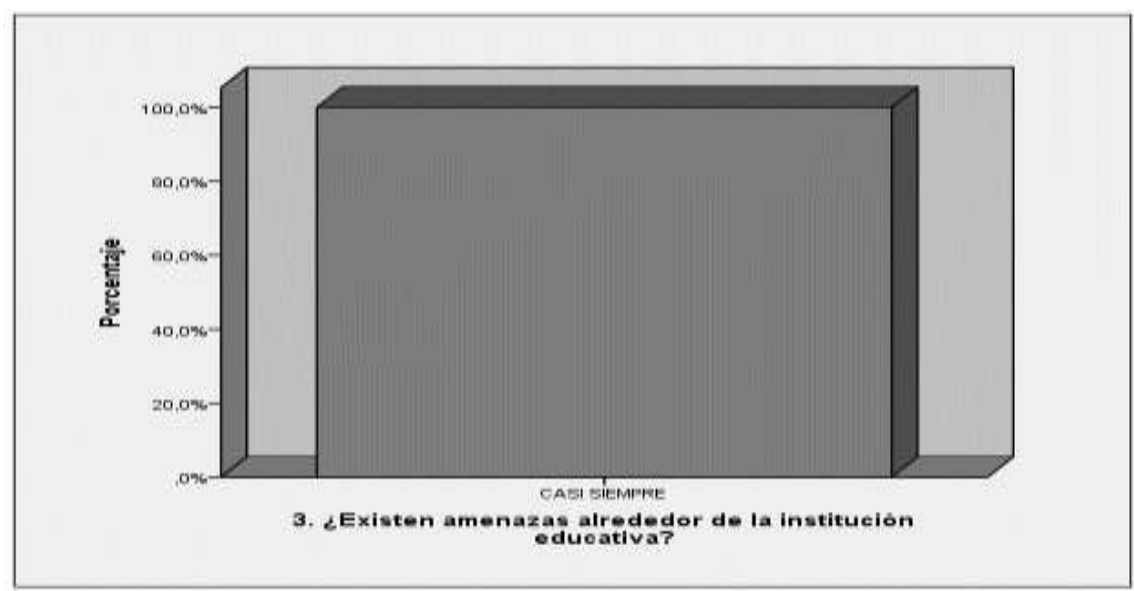

Interpretación: De acuerdo a la tabla estadística y el grafico, se evidencia un total de 2 Directivos encuestados representando que el 100,0\% manifiesta que casi siempre existen amenazas alrededor de la institución educativa. 
TABLA N $\mathbf{N}^{\circ} 05$

4. ¿Se busca oportunidades podrá lograr con éxito mediante las habilidades, capacidades y recursos con los que cuenta la institución?

\begin{tabular}{|l|l|l|l|l|l|}
\hline \multicolumn{2}{|l|}{} & Frecuencia & Porcentaje & $\begin{array}{l}\text { Porcentaje } \\
\text { válido }\end{array}$ & $\begin{array}{l}\text { Porcentaje } \\
\text { acumulado }\end{array}$ \\
\hline Válido & CASI SIEMPRE & 2 & 100,0 & 100,0 & 100,0 \\
\hline
\end{tabular}

Fuente: Datos procesados mediante el software SPSS 22 y Excel

Elaborado: Por la responsable de la investigación.

\section{GRÁFICO Nº4}

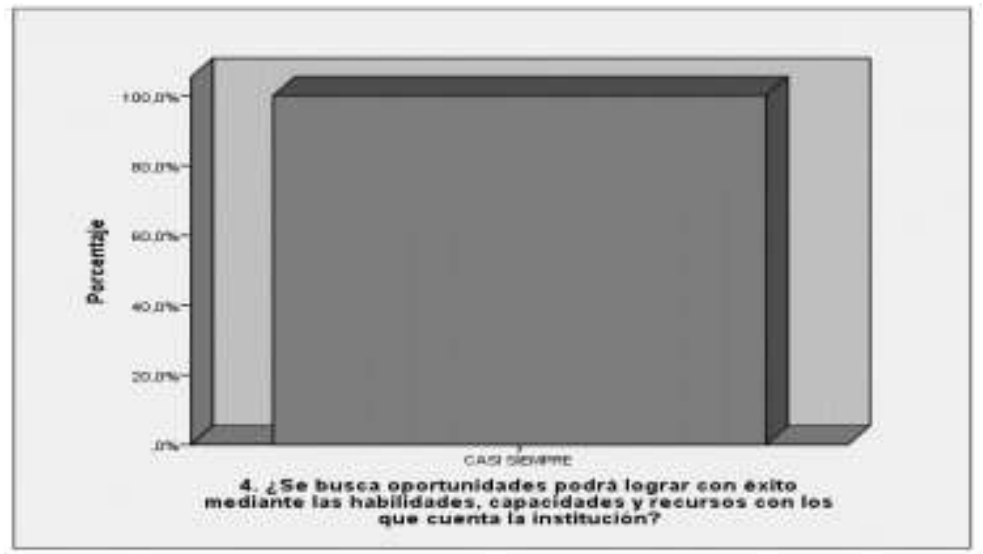

Interpretación: De acuerdo a la tabla estadística y el grafico, se evidencia un total de 2 Directivos encuestados representando que el 100,0\% manifiesta que casi siempre se busca oportunidades para lograr con éxito, mediante las habilidades, capacidades y recursos con los que cuenta la institución.

\section{TABLA Nº6}

\section{5. ¿Existen problemas estratégicos que enfrenta la institución?}

\begin{tabular}{|l|l|l|l|l|l|}
\hline \multicolumn{2}{|c|}{} & Frecuencia & Porcentaje & $\begin{array}{l}\text { Porcentaje } \\
\text { válido }\end{array}$ & $\begin{array}{l}\text { Porcentaje } \\
\text { acumulado }\end{array}$ \\
\hline \multirow{3}{*}{ Válido } & CASI SIEMPRE & 1 & 50,0 & 50,0 & 50,0 \\
\cline { 2 - 6 } & SIEMPRE & 1 & 50,0 & 50,0 & 100,0 \\
\cline { 2 - 6 } & Total & 2 & 100,0 & 100,0 & \\
\hline
\end{tabular}

Fuente: Datos procesados mediante el software SPSS 22 y Excel.

Elaborado: Por la responsable de la investigación.

\section{GRÁFICO N ${ }^{\circ} 05$}

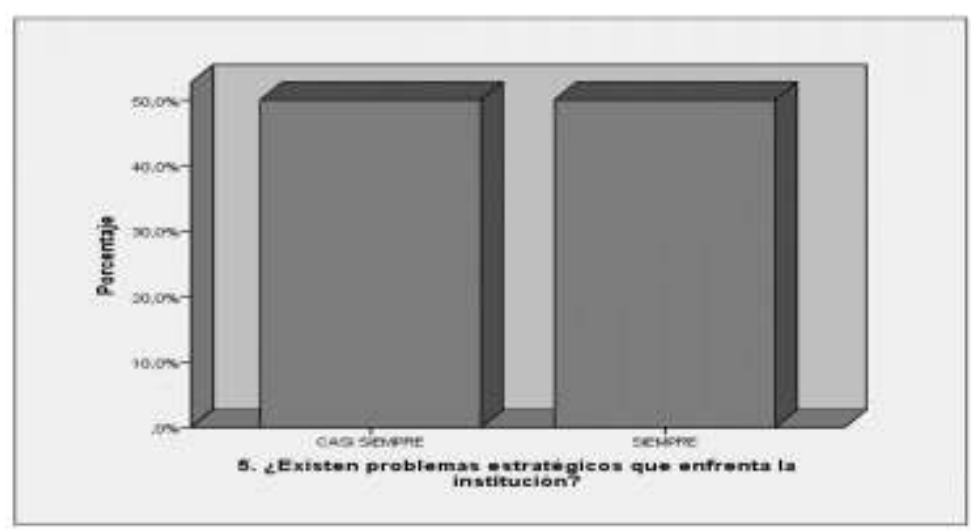




\section{Journal of the Academy $|165|$}

Interpretación: De acuerdo a la tabla estadística y el grafico, se evidencia un total de 2 Directivos encuestados representando que el 50,0\% manifiesta que siempre existen problemas estratégicos que enfrenta la institución y el 50,0\% señala que casi siempre.

\section{ORGANIZACIONAL}

\section{TABLA N ${ }^{\circ} 7$}

\begin{tabular}{|l|l|l|l|l|l|}
\hline \multicolumn{6}{|l|}{ ¿Elaboran, ejecutan y evalúan el reglamento interno? } \\
\hline \multirow{3}{*}{} & Frecuencia & Porcentaje & $\begin{array}{l}\text { Porcentaje } \\
\text { válido }\end{array}$ & $\begin{array}{l}\text { Porcentaje } \\
\text { acumulado }\end{array}$ \\
\hline \multirow{3}{*}{ Válido } & CASI SIEMPRE & 1 & 50,0 & 50,0 & 50,0 \\
\cline { 2 - 6 } & SIEMPRE & 1 & 50,0 & 50,0 & 100,0 \\
\cline { 2 - 6 } & Total & 2 & 100,0 & 100,0 & \\
\hline
\end{tabular}

Fuente: Datos procesados mediante el software SPSS 22 y Excel.

Elaborado: Por la responsable de la investigación.

\section{GRÁFICO No6}

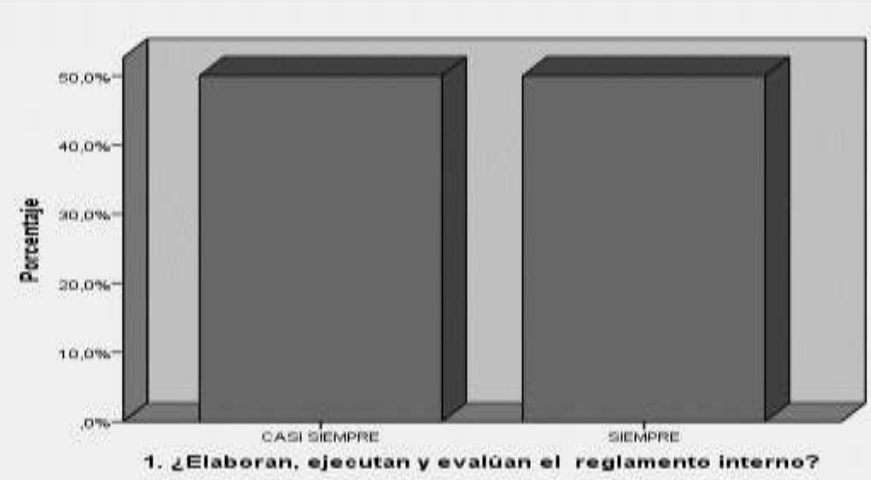

Interpretación: De acuerdo a la tabla estadística y el grafico, se evidencia un total de 2 Directivos encuestados representando que el 50,0\% manifiesta que casi siempre se elabora, ejecuta y evalúa el reglamento interno y el 50,0\% señala que siempre.

\section{TABLA N ${ }^{\circ} 8$}

\section{2. ¿Participan todos los integrantes de la institución en la elaboración de nuestro PEI?}

\begin{tabular}{|l|l|l|l|l|l|}
\hline \multicolumn{2}{|l|}{} & Frecuencia & Porcentaje & $\begin{array}{l}\text { Porcentaje } \\
\text { válido }\end{array}$ & $\begin{array}{l}\text { Porcentaje } \\
\text { acumulado }\end{array}$ \\
\hline Válido & CASI SIEMPRE & 2 & 100,0 & 100,0 & 100,0 \\
\hline
\end{tabular}

Fuente: Datos procesados mediante el software SPSS 22 y Excel.

Elaborado: Por la responsable de la investigación. 


\section{Journal of the Academy $|166|$}

\section{GRAFICO $\mathbf{N}^{\circ} 7$}

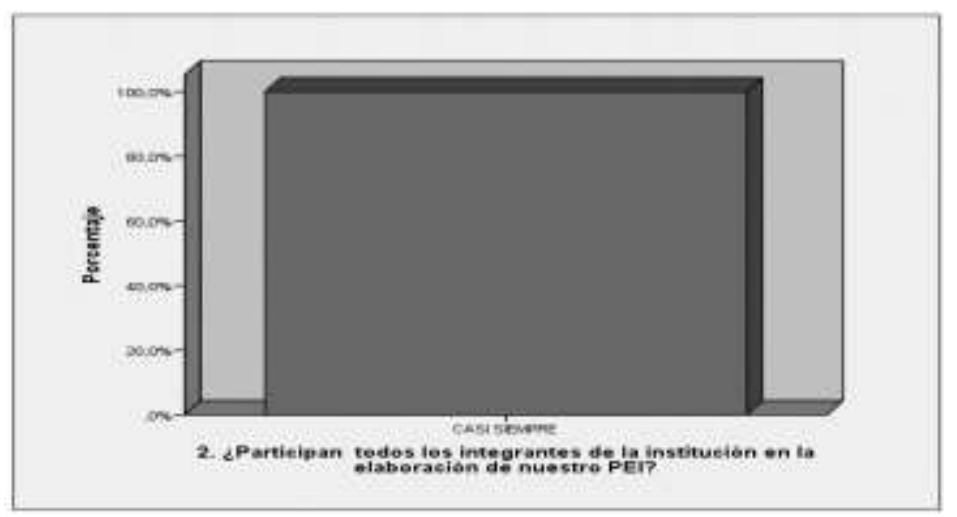

Interpretación: De acuerdo a la tabla estadística y el grafico, se evidencia un total de 2 Directivos encuestados representando que el 100,0\% manifiesta que casi siempre participan todos los integrantes de la institución en la elaboración de nuestro PEI.

\section{TABLA No9}

\begin{tabular}{|l|l|l|l|l|l|}
\hline \multicolumn{6}{|l|}{ 3. Participan activamente en la elaboración de nuestro PCI? } \\
\hline & Frecuencia & Porcentaje & $\begin{array}{l}\text { Porcentaje } \\
\text { válido }\end{array}$ & $\begin{array}{l}\text { Porcentaje } \\
\text { acumulado }\end{array}$ \\
\hline Válido & CASI SIEMPRE & 2 & 100,0 & 100,0 & 100,0 \\
\hline
\end{tabular}

Fuente: Datos procesados mediante el software SPSS 22 y Excel Elaborado: Por la responsable de la investigación.

\section{GRÁFICO N8}

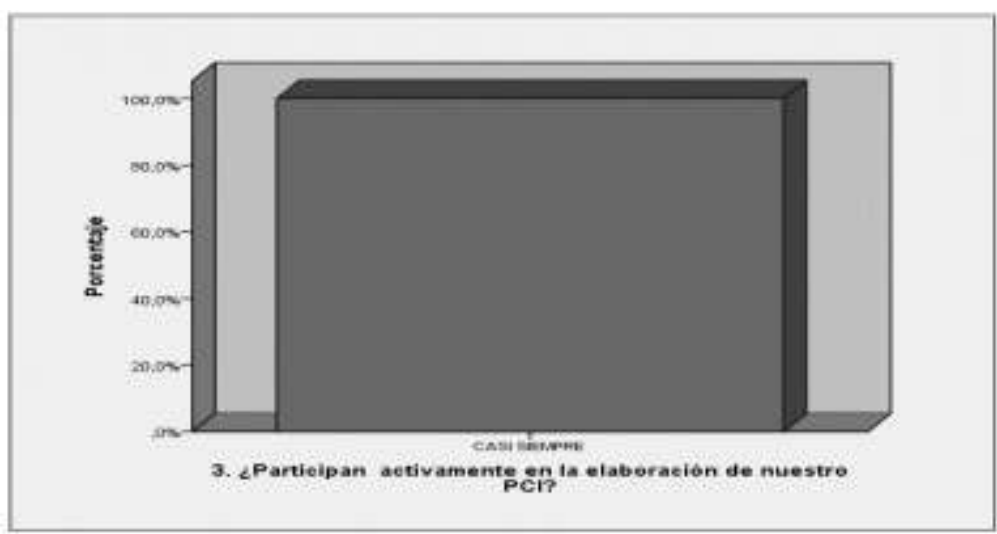

Interpretación: De acuerdo a la tabla estadística y el grafico, se evidencia un total de 2 Directivos encuestados representando que el 100,0\% manifiesta que casi siempre participan activamente en la elaboración de nuestro PCI.

\section{TABLA $\mathbf{N}^{\circ} 10$}

\begin{tabular}{|l|l|l|l|l|l|}
\hline \multicolumn{6}{|c|}{ 4.Se utiliza la ficha de monitoreo y luego de aplicarla se conversa con los docentes? } \\
\hline \multicolumn{2}{|c|}{} & Frecuencia & Porcentaje & $\begin{array}{l}\text { Porcentaje } \\
\text { válido }\end{array}$ & $\begin{array}{l}\text { Porcentaje } \\
\text { acumulado }\end{array}$ \\
\hline \multirow{3}{*}{ Válido } & CASI SIEMPRE & 1 & 50,0 & 50,0 & 50,0 \\
\cline { 2 - 6 } & SIEMPRE & 1 & 50,0 & 50,0 & 100,0 \\
\cline { 2 - 6 } & Total & 2 & 100,0 & 100,0 & \\
\hline
\end{tabular}


Fuente: Datos procesados mediante el software SPSS 22 y Excel.

Elaborado: Por la responsable de la investigación.

\section{GRÁFICO Nº}

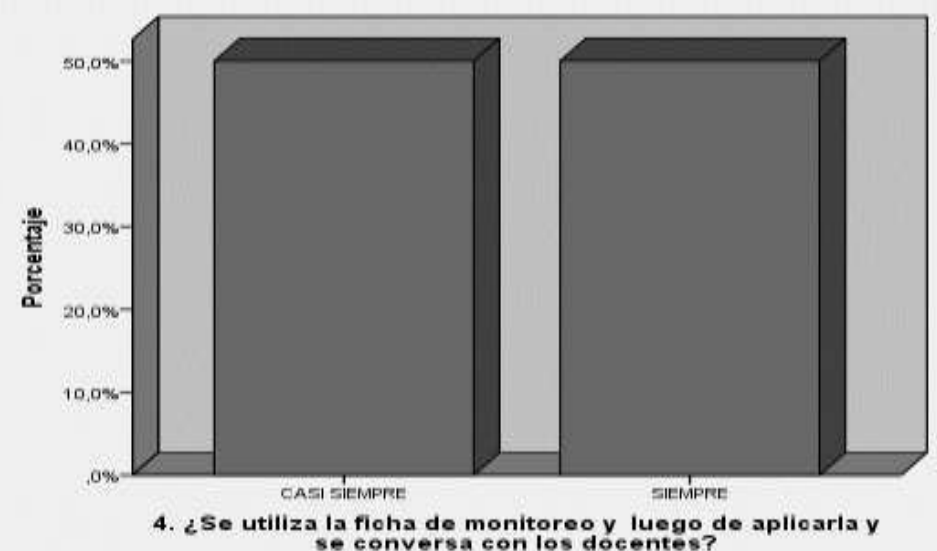

Interpretación: De acuerdo a la tabla estadística y el grafico, se evidencia un total de 2 Directivos encuestados representando que el 50,0\% manifiesta que casi siempre se utiliza la ficha de monitoreo y luego de aplicarla se conversa con los docentes y el 50,0\% señala que siempre.

\section{TABLA $\mathbf{N}^{\circ} 11$}

\begin{tabular}{|l|l|l|l|l|l|}
\hline \multicolumn{5}{|l|}{ 5. ¿Fue elegido en plenario los integrantes del CONEI y cumplen con sus funciones? } \\
\hline \multicolumn{2}{|l|}{} & Frecuencia & Porcentaje & Porcentaje válido & $\begin{array}{l}\text { Porcentaje } \\
\text { acumulado }\end{array}$ \\
\hline Válido & SIEMPRE & 2 & 100,0 & 100,0 & 100,0 \\
\hline
\end{tabular}

Fuente: Datos procesados mediante el software SPSS 22 y Excel.

Elaborado: Por la responsable de la investigación.

\section{GRÁFICO $\mathbf{N}^{\circ} 10$}

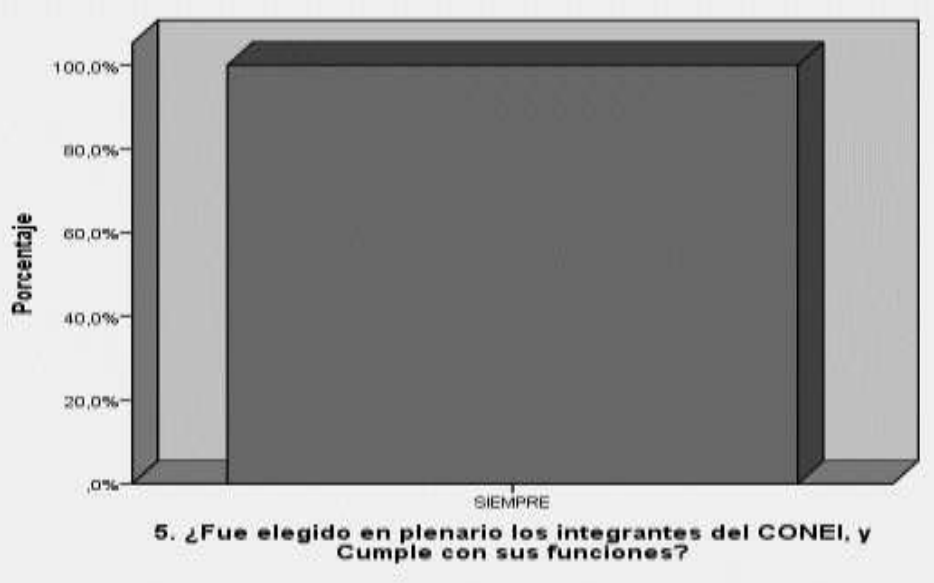

Interpretación: De acuerdo a la tabla estadística y el grafico, se evidencia un total de 2 Directivos encuestados representando que el 100,0\% manifiesta que siempre son elegidos en plenario los integrantes del CONEI y cumplen con sus funciones. 


\section{Journal of the Academy $|168|$}

\section{Discusión de resultados}

Después de analizado y contrastado las hipótesis, se ha realizado las siguientes discusiones sobre los resultados que a continuación se mencionan.

Los resultados estadísticos mostrados, para la hipótesis general de investigación la relación se cumple, pues si se realiza la técnica del FODA para una mejor organización entonces se realizara eficazmente una buena organización educativa con la investigación realizada por Campos (2014) quien aplicó un sistema de Planificación estratégica y control de Gestión en una Organización y permitió a la dirección facilitar la toma de decisiones en la implementación efectiva de planes y estrategias, de manera de mejorar su gestión, la cual coincide con los resultados vertidos en la investigación.

Confirmando los resultados con la teoría señalada por Bracho, Carruyo (2011) que determina la relación entre la planificación estratégica y la gestión del gerente educativo en instituciones de educación de acuerdo a los resultados indican que la aplicación de la planificación estratégica por parte de los gerentes educativos es una correlación muy alta.

Los resultados que obtuvo Díaz (2017) coinciden con los resultados de la presente investigación que indican que el 41,2\% de las instituciones educativas estatales, el nivel de calidad de la gestión educativa es regular.

Asimismo, señala Bautista (2017) en su estudio que el planeamiento estratégico si influye en el modelo de gestión basado en tres dimensiones: liderazgo asertivo, liderazgo pedagógico, liderazgo institucional. También define que se debe definir políticas, objetivos y lineamientos en el modelo de gestión que se desee implementar y formar parte del planeamiento estratégico, porque sería una norma a seguir como pauta de liderazgo en la gestión institucional.

\section{CONCLUSIONES}

Se determina que el FODA se relaciona eficazmente con la organización educativa de la Institución Educativa No 350202 "Zoila Amoretti de Odria, distrito Chaupimarca, Cerro de Pasco-2016, alcanzando una correlación significativa.

Asimismo, se recomienda establecer procesos bien definidos y elaborar manuales de procedimientos para cada ámbito de la gerencia educativa de la institución estudiada, para mejorar la calidad de la gestión educativa. Es necesario que la institución actualice su planificación estratégica considerando todos los ámbitos de la gerencia educativa, alineándose con los estándares de calidad educativa que establece el Ministerio de educación.

\section{REFERENCIAS BIBLIOGRÁFICAS}

Bautista Cruz, Antonio (2017) "Influencia del planeamiento estratégico en el modelo de gestión en las instituciones educativas de la "Provincia Franciscana de los XII Apóstoles" Lima - Perú 2015. Editorial: Universidad Nacional Mayor de San Marcos.

Bracho P. Kleeder \& Carruyo D. Norcelly (2011) "Planificación estratégica y gestión del gerente educativo en instituciones de educación primaria". Revista Praxis No. 7 ISSN: 1657-4915 Págs. 13 29 Santa Marta, Colombia.

Campos G. Fabián (2014) “Aplicación de un sistema de planificación estratégica y control de gestión en una organización sin fines de lucro: caso ONG psicólogos voluntarios" Universidad de Chile, Posgrado Economía y Negocios. 
Castro Sáez, Bernardo. (2001). LA ORGANIZACION EDUCATIVA: UNA APROXIMACION DESDE LA COMPLEJIDAD. Estudios pedagógicos (Valdivia), (27), 97-110. https://dx.doi.org/10.4067/S0718-07052001000100007

de Miguel Díaz, M. (1989). Modelos de investigación sobre organizaciones educativas. Revista de Investigación Educativa, 13(7), 21-57.

Dirección Regional de Educación de Pasco. (2015). Proyecto Educativo Regional de Pasco 2016-2021. Perú. Recuperado de https://www.drepasco.gob.pe/wp-content/uploads/2016/07/per-pasco-20162021.pdf

Díaz Vásquez, Selva (2017) Tesis "Calidad de la gestión educativa en el marco del proceso de la acreditación, en las instituciones educativas estatales nivel secundaria, zona urbana distrito de Iquitos 2016" para optar el grado de magister en educación con mención en gestión educativa Iquitos-Perú. Facultad de Ciencias de la Educación y Humanidades, Universidad Nacional de Iquitos.

López, T. G., \& Flores, M. C. (2013). El Foda: Una técnica para el analisis de problemas en el contexto de la planeacion en las organizaciones. Recuperado de https://www.uv.mx/iiesca/files/2013/01/foda1999-2000.pdf

Power, D.J, Gannon, M.J., McGinnis, M.A. and Schweiger, D.M. (1986): Strategic Management Skills. Reading, MA, Addison Wesley, USA, citado en Lazzari y Maesschaclk (2002). Recuperado de https://dialnet.unirioja.es/servlet/articulo?codigo $=2469967$

Reales Chacón, Lisbeth Josefina, \& Arce Aguirre, Jacinto Antonio, \& Heredia Gómez, Frank Adolfo (2008). LA ORGANIZACIÓN EDUCATIVA Y SU CULTURA: UNA VISIÓN DESDE LA POSTMODERNIDAD. Laurus, 14(26),319-346.[fecha de Consulta 3 de Junio de 2020]. ISSN: 1315883X. Disponible en: https://www.redalyc.org/articulo.oa?id=761/76111491016

Salas, L., Marco Salas, and Beatriz Herrera. (2011) "Importancia del liderazgo escolar para la consecución de la calidad en instituciones de educación media superior." Cuadernos de educación y desarrollo 3.29: 1-1. 